\title{
Colgajo pediculado en filete de extremidad inferior para cierre de úlceras por presión complejas y complicadas
}

\author{
Pedicled fillet of leg flap for closure of complex and complicated pressure sores
Dr. Raúl Servando Caracheo-Rodríguez,* Dra. Cecilia González-Martínez, ${ }^{\ddagger}$ Dr. Óscar Alejandro Sánchez-García ${ }^{\ddagger}$

\begin{abstract}
Palabras clave: Colgajos en filete, partes de repuesto, úlceras por presión
\end{abstract} complejas.

Keywords: Fillet flaps, spare parts, complex pressure sores.
${ }^{*}$ Cirujano Plástico y Reconstructivo. Departamento de Cirugía Plástica y Reconstructiva.

${ }^{\ddagger}$ Médico Residente de Cirugía General.

Hospital General de Querétaro, Secretaría de Salud del Estado de Querétaro (SESEQ).

Querétaro, Querétaro, México.

Los autores de este artículo no tienen conflicto de intereses que declarar.

Recibido:

20 julio 2019

Aceptado para publicar:

15 enero 2020

\section{RESUMEN}

Las úlceras por presión múltiples (complejas), recurrentes (complicadas) o extensas representan un reto reconstructivo para el cirujano plástico. En este artículo, se presenta el caso de un paciente del sexo masculino de 20 años, que tenía como antecedentes paraplejia de nueve años de evolución con presencia de múltiples úlceras de grado IV. Se realizó un colgajo total de la extremidad inferior en filete mediante la técnica de Jandali, logrando cubrir las lesiones en su totalidad con una adecuada evolución del colgajo en los días posteriores. El colgajo en filete sigue siendo de utilidad y es un método confiable para la reconstrucción de úlceras complejas por presión.

\section{ABSTRACT}

Multiple (complex) pressure sores, recurrent (complicated) or large, represent a reconstructive challenge to the plastic surgeon. We present the case of a 20-year-old male patient with a history of paraplegia of nine years of evolution with multiple IV grade ulcers. A total leg flap fillet was performed using the Jandali technique, and the lesions were completely covered, with an adequate evolution of the flap in the follow days. The leg flap fillet remains a useful and reliable method for complex pressure sores reconstruction.

\section{INTRODUCCIÓN}

L as úlceras por presión son lesiones isquémicas que se localizan en la piel y los tejidos subyacentes $y$, en general, se producen donde se localiza una prominencia ósea. Este tipo de úlceras constituyen un problema recurrente y generalizado en pacientes que presentan paraplejia o cuadriplejia a consecuencia de una lesión medular. En estos casos, para cubrir defectos de tamaño modesto, se han utilizado con frecuencia diversos colgajos locales y regionales procedentes del glúteo y muslo; sin embargo, cuando las úlceras de grado IV se tornan recurrentes o extensas, esos colgajos pueden ya no estar disponibles o ser insuficientes.

De igual manera, puede haber reincidencia de las úlceras por un mal manejo postopera- torio o de rehabilitación, lo que condiciona la formación de nuevas úlceras donde ya se había realizado la reconstrucción y, por lo tanto, una pérdida catastrófica de la oportunidad quirúrgica del colgajo realizado y ahora perdido. ${ }^{1-3}$ La presencia de múltiples úlceras en el mismo paciente o de úlceras recidivantes en pacientes ya reconstruidos añade a su definición los términos de «compleja o complicada». El tejido de una extremidad amputada o no salvable puede ser utilizado para la reconstrucción de estas lesiones complejas o complicadas. Éste es el concepto de «partes de repuesto», considerando que una de sus principales ventajas es que no van a aumentar la morbilidad del sitio donador.

Los colgajos en filete (o fillet flaps) se utilizan con frecuencia bajo este principio y se definen 
como colgajos compuestos de patrón axial, que pueden ser transferidos de manera libre o pediculada y pueden proveer piel, músculo, fascia y hueso. ${ }^{1}$ Estos colgajos se pueden utilizar en la reconstrucción de defectos mayores causados por la resección tumoral o para el tratamiento de úlceras de presión recurrentes; en estas últimas, el colgajo es levantado de una extremidad intacta adyacente y representa una solución alterna a la microcirugía, o cuando ha fracasado una reconstrucción previa o existen pocas opciones viables. Estas situaciones son raras y la ventaja de la cobertura con colgajos en filete debe superar la pérdida de la extremidad. ${ }^{2}$ En este artículo, a continuación se presenta el caso clínico de un paciente en quien se usó el uso del colgajo total en filete de extremidad inferior para la cobertura de úlceras por presión complicadas.

\section{CASO CLÍNICO}

Se trata de un paciente del sexo masculino de 20 años, con antecedente de paraplejia de nueve años de evolución consecuencia de una herida por arma de fuego. A su ingreso, presentaba múltiples úlceras por presión grado IV, sin datos de infección, con úlcera trocantérica e isquiática izquierdas, dislocación de la articulación de la cadera y úlceras sacra e isquiática derecha.

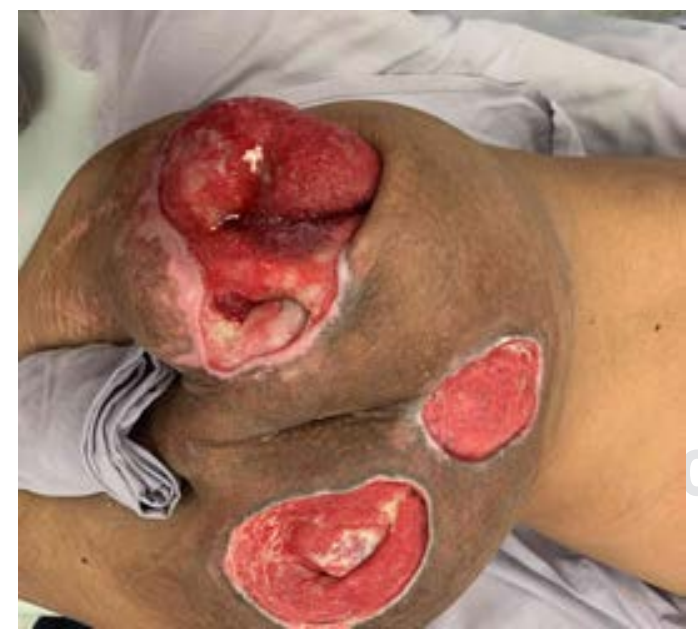

Figura 1: Paciente con úlcera trocantérica e isquiática izquierdas, dislocación de la articulación de la cadera y úlceras sacra e isquiática derecha.

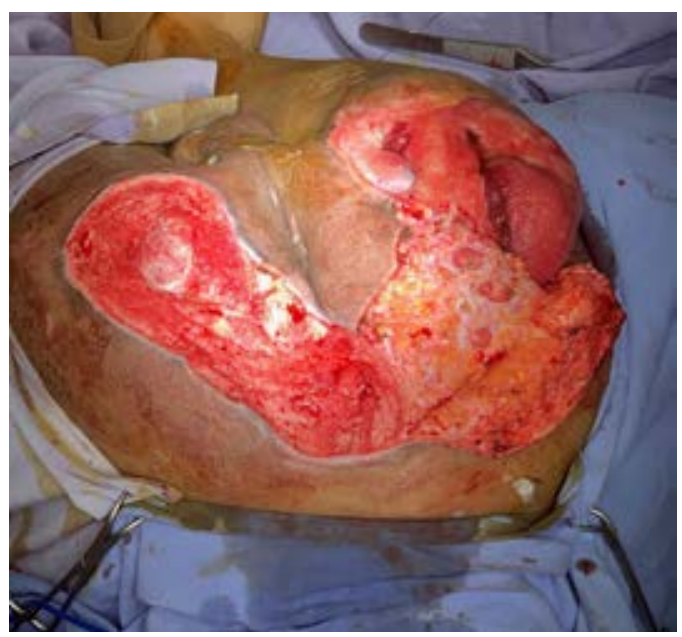

Figura 2: Se retiraron los puentes de piel entre las úlceras trocantérica, sacra y biisquiática y quedó un área de aproximadamente $500 \mathrm{~cm}^{2}$.

El paciente había sido reconstruido en una ocasión con un colgajo Hamstring para úlcera isquiática izquierda, del que se apreciaba la cicatriz (Figura 1). Durante su estancia en el hospital, fue manejado con apoyo nutricional, desbridamiento y curaciones de las lesiones, así como control de infecciones del tracto urinario; además, se le realizó una colostomía terminal como medida de control de la constante contaminación fecal de las lesiones. Se propuso manejo quirúrgico definitivo mediante la realización de colgajo total de extremidad inferior izquierda. A continuación se describe la técnica quirúrgica.

\section{Técnica}

Se realizó el procedimiento tal y como lo describió Jandali en 2009. ${ }^{2}$ Es decir, en nuestro caso, cuando el paciente se encontraba en posición de navaja sevillana, debido a la contractura de la cadera y rodilla que tenía (Figura 1), se le realizó asepsia y antisepsia, usando una solución de clorhexidina al 2\%. Posteriormente, se retiraron los puentes de piel entre las tres lesiones (trocantérica, sacra y biisquiática), dejando una única lesión de gran tamaño de aproximadamente $500 \mathrm{~cm}^{2}$ (Figura 2). Entonces un primer equipo quirúrgico resecó el tejido de granulación excesivo hasta encontrar el plano 


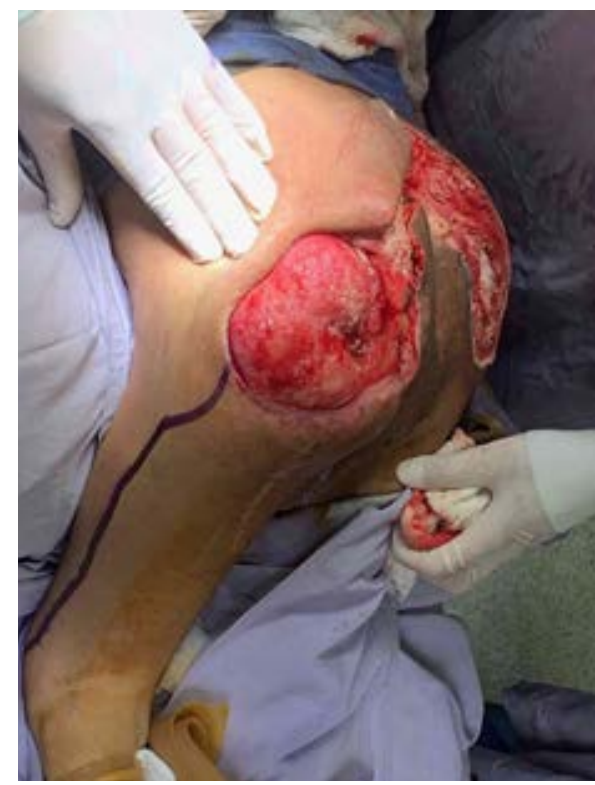

Figura 3: Marcaje de la incisión lateral del colgajo del miembro pélvico izquierdo.

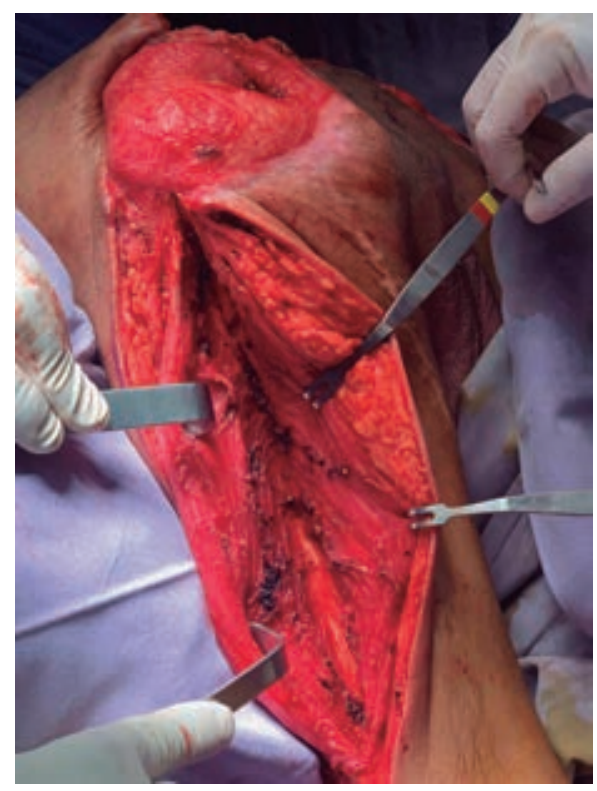

Figura 4: Separación de los músculos cuádriceps (vasto lateral) e isquiotibiales para una rápida exposición del fémur.

muscular y se procedió a levantar el colgajo del miembro pélvico izquierdo por medio de una incisión lateral (Figura 3), entre los músculos cuádriceps (vasto lateral) e isquiotibiales, teniendo una rápida exposición del fémur, el cual se denudó con facilidad en el plano subperióstico con un disector (Figura 4).

El segundo equipo quirúrgico realizó la amputación del pie a la altura de la rodilla, con isquemia, para permitir simultáneamente la re-

Figura 5:

Exposición de la tibia donde se muestra la membrana interósea con los vasos tibiales anteriores.
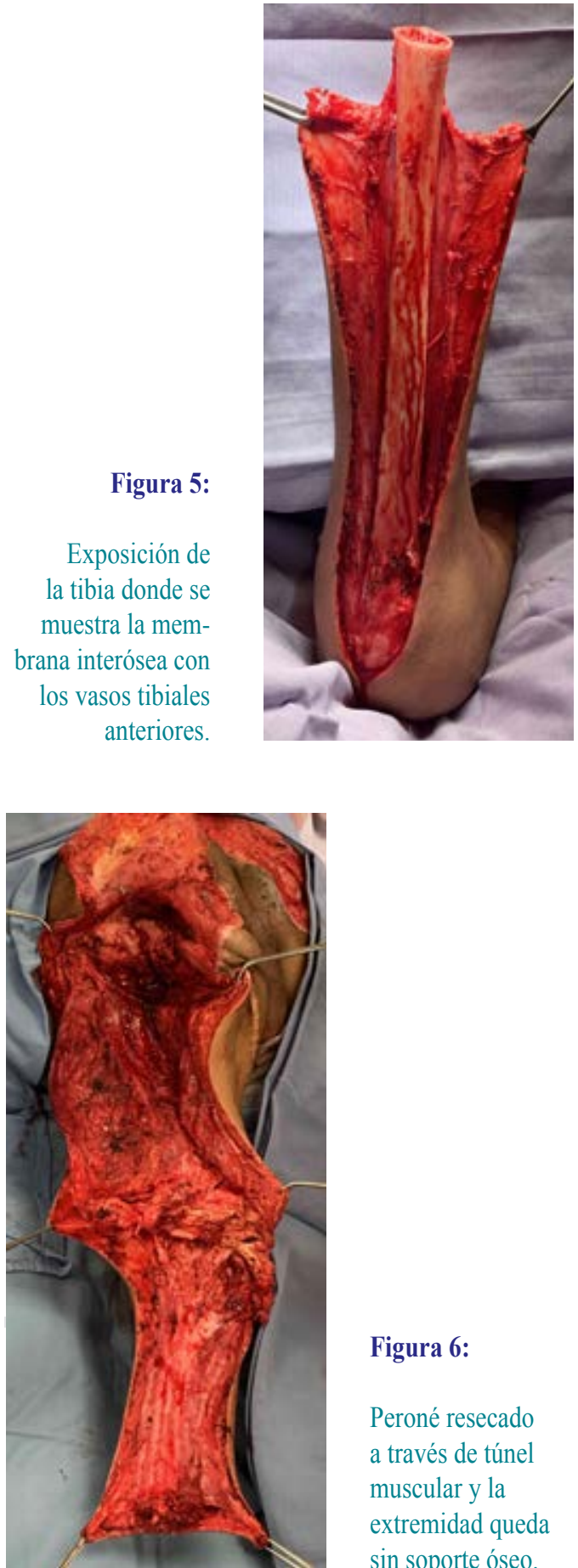

Figura 6:

Peroné resecado a través de túnel muscular y la extremidad queda sin soporte óseo. 
sección del fémur. El pie se amputó de manera circunferencial por arriba del tobillo; las arterias tibiales anteriores y posteriores y los vasos peroneos se identificaron y ligaron con seda 3-0. Con el objetivo de facilitar el trabajo de los dos equipos, se realizó un corte en la porción medial del fémur. La incisión lateral del muslo se continuó lateral a la rodilla y de allí se medializó para pasar hacia anterior, transversal a la rótula y, posteriormente, hacia distal, sobre la tuberosidad de la tibia, y se continuó sobre su borde anterior, hasta llegar al extremo donde se amputó el pie. La exposición de la tibia se realizó con un desperiostizador, teniendo cuidado de no lesionar la membrana interósea para preservar el flujo de los vasos tibiales anteriores (Figura 5). La rótula se extrajo abriendo el tendón del cuádriceps, se retiraron los meniscos y la tibia fue removida después de ser separada de la cabeza del peroné.

El peroné se accedió a través de un túnel submuscular, en el que tanto la cabeza del peroné y la parte distal se diseccionaron en forma subperióstica, utilizando para ello un elevador. El peroné se resecó a través del túnel muscular, dejando la extremidad sin soporte óseo alguno (Figura 6). Asimismo, se resecó el fémur proximal, encontrando luxada la articulación de la cadera, por lo que se extrajo la pieza con facilidad (Figura

\section{Figura 7:}

Miembro inferior izquierdo resecado;

la articulación de la cadera luxada facilitó la extracción de la pieza.

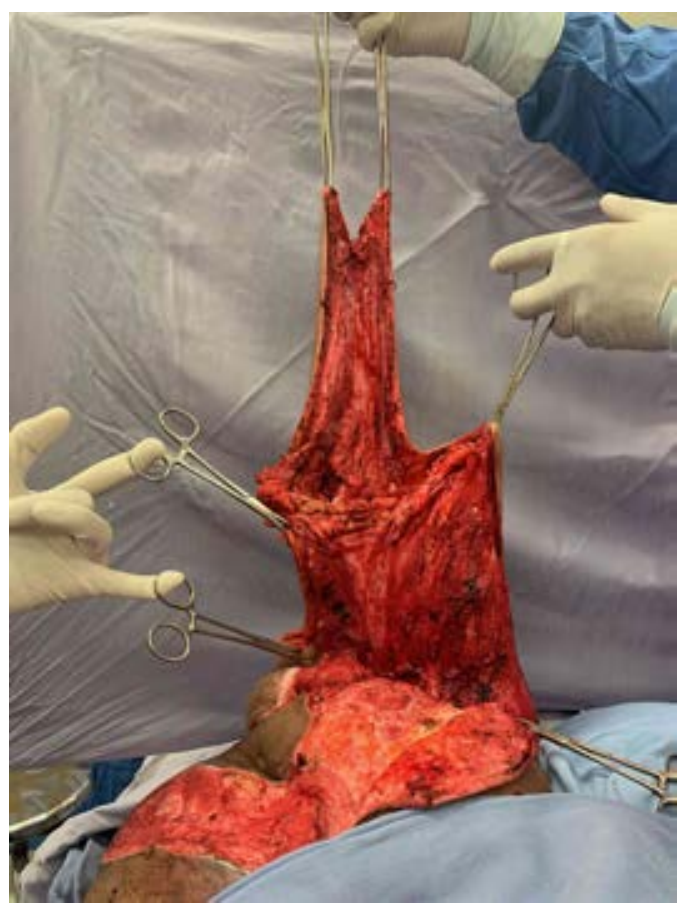

Figura 8: Colgajo levantado.

7). Se rotó el colgajo, logrando una cobertura total del defecto; se fijaron los músculos del colgajo al plano muscular del defecto para obliterar los espacios muertos. Se colocaron múltiples drenajes tipo Penrose y se cerró el tejido celular subcutáneo con vicryl 2-0 y la piel con puntos simples de nylon 2-0 (Figuras 8 a 10); el sangrado cuantificado durante el evento quirúrgico fue de $800 \mathrm{~mL}$. El momento quirúrgico de mayor pérdida sanguínea fue durante la disección del fémur proximal y la resección del tejido de la úlcera.

El paciente requirió transfusión de dos paquetes globulares en el postoperatorio inmediato, así como manejo del dolor por osteocondritis esternocostal (Tietze), debido a la posición ventral prolongada durante el evento quirúrgico, la cual remitió en los días subsiguientes. El manejo se completó con antibióticos vía intravenosa, medidas higiénicas y fisioterapia.

En el seguimiento a mediano y largo plazo, aunque el paciente era más bien indisciplinado y poco apegado a las medidas higiénicas y cuidados del colgajo después de la cirugía, él mismo soportó muy bien esos descuidos, teniendo solamente complicaciones menores, como una dehiscencia parcial de $4 \mathrm{~cm}$ en el 


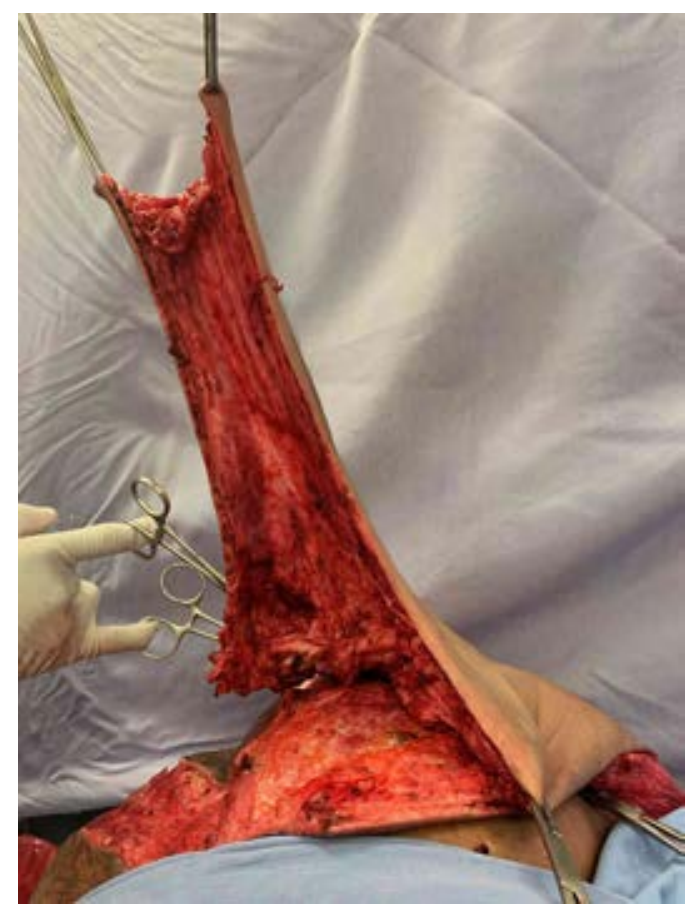

Figura 9: Colgajo presentado en el defecto.

borde superior. El paciente se mantenía recargado completamente sobre el colgajo por tiempos prolongados al permanecer sentado en su silla de ruedas y con frecuente movilización por deslizamiento, con el consiguiente cizallamiento del colgajo sobre la cresta iliaca posterior, así como de una pequeña zona deprimida en el borde interno del colgajo donde hubo una discreta separación de los labios de la herida, sin embargo, ya epitelizada, fueron suturadas en una ocasión y posteriormente se permitió el cierre secundario de la misma zona, por la dificultad para el transporte del paciente al centro hospitalario donde se realizó la reconstrucción.

Al cuarto y quinto mes después del cierre, se observó un adecuado colchón en la zona isquiática que se ha mantenido perenne a pesar del uso rudo que le ha dado el paciente, sólo manifestándose una pequeña zona cruenta en la parte superior (Figuras 11 y 12).

\section{DISCUSIÓN}

El caso como el que presentamos es escaso en la literatura mundial, si bien el concepto de «partes de repuesto» ha acompañado al cirujano plástico desde hace décadas y es parte sustancial del pensamiento reconstructivo; en éste, se busca aprovechar una extremidad no salvable para reparar lo que sí es salvable y brindar así el máximo beneficio. Como una consecuencia natural del concepto de «partes de repuesto», se encuentra el colgajo en filete (o fillet flap), y en él es necesario considerar en cada algoritmo del manejo del trauma para evitar una mayor «morbilidad del sitio donador». ${ }^{2}$

En 1956, Georgiade, ${ }^{4}$ y posteriormente, con un artículo complementario en 1969, Royer y colaboradores ${ }^{5}$ describieron el uso del colgajo total de muslo como tratamiento definitivo para úlceras trocantéricas y sacras grandes, dando inicio a los conceptos antes expuestos. Por su parte, Berkas y su equipo ${ }^{6}$ reportaron en 1961 una técnica similar en la que usaron tejido de hasta $5 \mathrm{~cm}$ por debajo de la rodilla para cubrir defectos sacros. No fue hasta 2009 cuando Jandali y su grupo de investigación ${ }^{2}$ publicaron el uso del colgajo total de extremidad inferior para el cierre de áreas cada vez más grandes; este colgajo lo realizaron en cuatro pacientes similares al que presentamos: con úlceras trocantéricas y sacras conjuntas, o isquiáticas con trocantéricas y sacras, o la combinación de ellas, en defectos de 650 hasta $800 \mathrm{~cm}^{2}$. Es curioso que este autor haya sido el primero en agregar a la discusión un aspecto etimológico

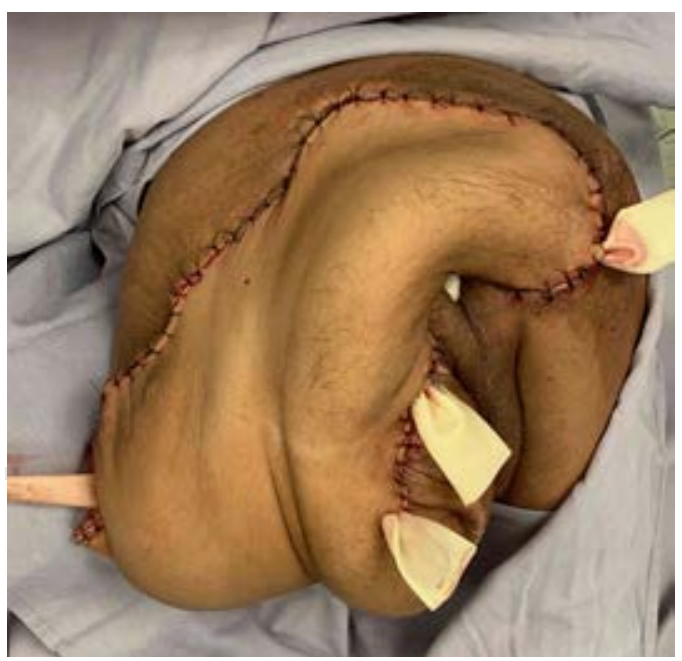

Figura 10: La rotación del colgajo logra la cobertura total del defecto, con drenajes tipo Penrose. 


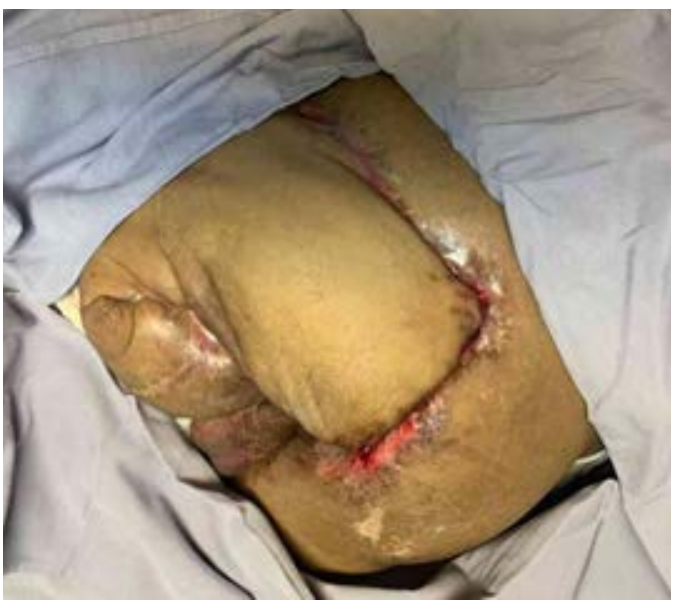

Figura 11: Cuarto mes postoperatorio. Se observa dehiscencia parcial del colgajo.

interesante en la definición del colgajo en filete: pues, como el nombre lo dice, alude a un corte de carne o de pescado sin hueso, aspecto que no mencionan otros autores, o lo consideran redundante para mencionarlo.

En 2014 volvió a aparecer este colgajo en la literatura con el escrito de Verveld y otros, ${ }^{7}$ que aunque tenía variaciones en la técnica, funcionaba bajo el mismo principio; en ese caso, tunelizaron el colgajo para llevarlo a la zona problema. En 2019, apareció otra vez en una publicación de Georgiou, ${ }^{8}$ en un caso de un paciente obeso mórbido con úlceras trocantéricas, sacra e isquiática, variando de nuevo la técnica al hacer todo el acceso desde el plano posterior del muslo, la rodilla y la pierna, lo que consideramos de mayor dificultad técnica que la descrita originalmente por Jandali.

En una revisión hasta 2016, realizado por McCarthy, ${ }^{9}$ sólo había siete artículos que se referían a los colgajos en filete parciales o totales de la extremidad inferior para el cierre de úlceras de presión; sin embargo, en nuestra revisión, la cual abarca hasta 2019, solamente hay tres autores que utilizan prácticamente la totalidad de la extremidad inferior para el cierre de las úlceras complejas ${ }^{2,7,8}$ que, como norma, abarcan úlceras de ambos lados de la línea media, es decir, coexisten úlceras derechas e izquierdas en el mismo paciente. Por lo tanto, se requiere de un colgajo que cubra la máxima longitud del defecto, usando como punto-pivote una de las caderas para alcanzar la otra. Hasta donde revisamos, este artículo es apenas el cuarto en la literatura mundial y el primero en nuestro país que menciona esta técnica.

Aunque parece un manejo radical $-y$ lo es-, este tipo de colgajos ofrece una forma relativamente segura y efectiva para el tratamiento de úlceras complejas o complicadas en las que los colgajos locales ya no son una opción; además, provee una adecuada obliteración del espacio muerto, restaura el colchón pélvico y brinda una superficie de piel adecuada.

Las indicaciones para este colgajo incluyen:

- Múltiples úlceras por decúbito o úlceras recurrentes con insuficiente tejido adyacente para una adecuada cobertura.

- Úlceras demasiado grandes para su reconstrucción mediante rotación de colgajo.

- Múltiples úlceras asociadas con contractura de cadera o rodilla.

- Úlceras con extensa osteomielitis de la pelvis. ${ }^{9}$

- Úlceras derechas e izquierdas coexistentes.

En el caso de nuestro paciente, solamente no tenía el penúltimo criterio. También, coincidiendo con varios de los autores mencionados, la principal complicación transoperatoria que

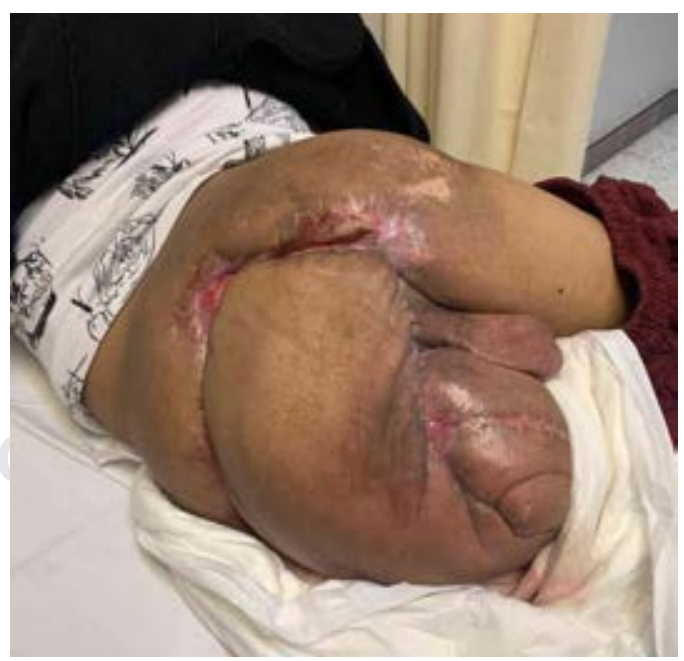

Figura 12: Quinto mes postoperatorio. Se aprecia un adecuado colchón en la zona isquiática. 
puede presentar esta técnica es la pérdida sanguínea, la cual puede ser manejada con facilidad con la adecuada previsión. En nuestro caso, no se presentó necrosis del colgajo en ningún estado postoperatorio y la cirugía no afectó la capacidad del paciente para su movilidad residual en cama o en silla de ruedas, incluso el paciente refirió mayor comodidad y menor esfuerzo de él o de sus familiares para la rehabilitación y cuidados postoperatorios, lo que refuerza la idoneidad y bondad del colgajo presentado.

\section{CONCLUSIONES}

Las úlceras por presión múltiples, complejas o complicadas representan un reto para el cirujano reconstructor. El colgajo pediculado en filete de la extremidad inferior es un colgajo grande y robusto que aprovecha sus dimensiones para alcanzar zonas extensas desde su punto-pivote, brindando además un adecuado colchón y piel de calidad, al tiempo que oblitera grandes espacios muertos, lo cual no aumenta la morbilidad del sitio donador. También, en forma subjetiva, brinda mejoría al paciente al permitirle una mejor movilidad residual y rehabilitación. Es un colgajo seguro y su uso es invaluable cuando se han agotado otras opciones locales, o la zona es muy extensa para cubrirla con colgajos.

\section{REFERENCIAS}

1. Küntscher MV, Erdmann D, Homann H, Steinau H, Levin SL, German G. The concept of fillet flaps: classification, indications, and analysis of their clinical value. Plast Reconstr Surg 2001; 108: 885-896.

2. Jandali S, Low DW. Pedicled fillet of leg flap for extensive pressure sore coverage. Eplasty 2009; 9: e50.

3. Lari AR, Rajacic N. One-stage repair of multiple bed sores. Br J Plast Surg 1992; 45: 540-543.

4. Georgiade N, Pickrell K, Maguire C. Total thigh flaps for extensive decubitus ulcers. Plast Reconstr Surg 1956; 17: 220-225.

5. Royer J, Pickrell K, Georgiade N. Total thigh flaps for extensive decubitus ulcers. A 16 year review of 41 total thigh flaps. Plast Reconstr Surg 1969; 44:109-118.

6. Berkas EM, Chesler MD, Sako Y. Multiple decubitus ulcer treatment by hip disarticulation and soft tissue flaps from the lower limbs. Plast Reconstr Surg 1961; 27: 618-619.

7. Verveld CJ, Fuchs SP, Buncamper MF, Winters H. The tunneled lower leg fillet flap, a reconstructive salvage option in patients with severe pressure ulcers. J Plast Reconstr Aesthet Surg 2014; 67: 427-428.

8. Georgiou I, Kruppa P, Ghods M. Use of a total leg fillet flap to cover multiple pelvic pressure ulcers. Plast Reconstr Surg Glob Open 2019; 7: e2084.

9. McCarthy JE, Venkat KR. Systematic review and operative technique of recalcitrant pressure ulcers using a fillet flap technique. Plast Reconstr Surg Glob Open 2016; 4: e1001.

\section{Correspondencia:}

\section{Dr. Raúl Servando Caracheo-Rodríguez}

Av. 5 de Febrero Núm. 101,

Col. Los Virreyes, 76170,

Santiago de Querétaro, Qro., México.

E-mail: servandocaracheo@hotmail.com 